\title{
Constraining cutoff physics in the cosmic microwave background
}

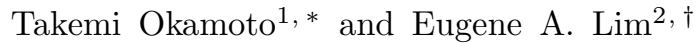 \\ ${ }^{1}$ The Department of Physics and the Center for Cosmological Physics, University of Chicago, Chicago, IL 60637 \\ ${ }^{2}$ The Department of Astronomy and Astrophysics and the Center \\ for Cosmological Physics, University of Chicago, Chicago, IL 60637
}

\begin{abstract}
We investigate the ability to constrain oscillatory features in the primordial power spectrum using current and future cosmic microwave background observations. In particular, we study the observability of an oscillation arising from imprints of physics at the cutoff energy scale. We perform a likelihood analysis on the WMAP data set, and find that the current data set constrains the amplitude of the oscillations to be less than 0.77 at $2 \sigma$, consistent with a power spectrum without oscillations. In addition, we investigate the fundamental limitations in the measurement of oscillation parameters by studying the constraints from a cosmic variance limited experiment. We find that such an experiment is capable of constraining the amplitude of such oscillations to be below 0.005 , implying that reasonable models with cutoff energy scales $\Lambda>200 H_{\text {inf }}$ are unobservable through the microwave background.
\end{abstract}

\section{INTRODUCTION}

Recent observations of cosmic microwave background (CMB) anisotropies by the Wilkinson Microwave Anisotropy Probe (WMAP) 1, 2] have raised the possibility of determining the initial conditions for structure formation with high precision. The most recent data are consistent with a power-law primordial power spectrum, as predicted by most variants of the inflationary paradigm 3, 4, 5, 6]. At the same time, marginal detections of a running of the power-law index, as well as glitches in the temperature power spectrum across several multipoles, suggest that the primordial power spectrum may deviate from the power-law form, and the presence of such features may be tested with future experiments such as the Planck satellite [7] and CMBPol 8].

Previous works have taken model independent approaches to the measurement of features in the power spectrum. These parametrize the primordial power spectrum in band powers or wavelets [9, 10, 11, 12], or choose a set of window functions to regularize the initial power spectrum [13, 14]. An alternate, observation-oriented approach involves constructing the principal components of the primordial power spectrum, which are guaranteed to be the modes that best constrain deviations from the power-law form 15. Such general methods are ideal for situations where the deviations from the power-law form are unknown. However, if a well-motivated form for the deviations exists, the constraints can be improved by incorporating the specific form in the analysis.

A form of modification that is favored by several mechanisms is that of an oscillation on top of the usual power-law form. Although such a modification can arise from interactions of the inflaton with other fields [16], we specifically consider the modifications that arise from an ambiguity in the vacuum choice at some high-energy cutoff scale. The presence of a high-energy cutoff in the description of fluctuations during inflation naturally leads to an oscilatory modification 17, 18, 19, 20, 21. Current data sets allow a very loose limit on a restricted, two-parameter form of the oscillation [22], and an oscillation may be favored [23], although no constraints have been derived on the parameters that define the oscillations. In addition, there is some disagreement over whether such effects might be observable by future experiments, and parameter forecasts are absent from current literature [22, 24]. Furthermore, the previous studies do not account for the full, four-parameter phenomenology that parametrizes our ignorance of the physics at the cutoff scale.

In this work, we quantify the limit on such modifications by using current CMB data to constrain the parameters that define the oscillations. Furthermore, we provide robust forecasts for the oscillation parameters by considering the constraints from a cosmic variance limited experiment. We briefly review the theoretical framework in Sec. III and describe the statistical method in Sec. [II] We present the bounds from current CMB data in Sec. [V] and the limitations due to cosmic variance in Sec. D] We conclude in Sec. VI]

\footnotetext{
*Electronic address: tokamoto@oddjob.uchicago.edu

${ }^{\dagger}$ Electronic address: elim@jasmine.uchicago.edu
} 


\section{THEORETICAL FRAMEWORK}

In the inflationary scenario, inhomogeneities in the matter density arise due to quantum fluctuations of the inflaton, the scalar field that induces inflation. Computations in such a scenario assume that the quantum field theoretic description of the inflaton remains valid to arbitrarily small length scales; however, we expect quantum field theory to break down at some length scale, so that a complete description of the quantum fluctuations would have to incorporate the unknown physics at the cutoff scale.

In the absence of information concerning the nature of the high-energy cutoff, we assume that the effects of any physics beyond the cutoff is equivalent to ambiguities in the choice of vacuum at the cutoff scale. We describe a model that parametrizes our ignorance about the cutoff physics, and briefly review the relation between the primordial power spectrum from inflation and the cosmic microwave background.

\section{A. Deviations from scale invariance}

In this section we briefly review the theory of perturbation generation in an inflationary spacetime, and describe a generic model for deviations from a scale-invariant power spectrum. A complete treatment can be found in Ref. 21]. We follow the notation in Ref. [21] except for a change in metric signature.

We begin by considering a theory of inflation with a minimally coupled scalar field with a potential $V(\phi)$

$$
S=\int d^{4} x \sqrt{-g}\left[\frac{m_{p}^{2}}{2} R-\frac{1}{2}(\partial \phi)^{2}-V(\phi)\right],
$$

where $m_{p}^{2} \equiv(8 \pi G)^{-1}$ is the reduced Planck mass. Since the universe is spatially homogenous and isotropic we will use the Robertson-Walker metric:

$$
d s^{2}=a^{2}(\eta)\left(-d \eta^{2}+\delta_{i j} d x^{i} d x^{j}\right)
$$

Note that the conformal time $\eta$ is taken to run from $-\infty$ to 0 . We assume a spatially flat metric for simplicity.

If, for a certain range of $\phi$, the potential $V(\phi)$ is nearly constant and thus the field is rolling slowly, then the scalar field behaves like a cosmological constant and the universe inflates during this phase. In the slow-roll limit, the potential can be characterized by the parameters [25]

$$
\begin{aligned}
\epsilon & \equiv \frac{3 \phi^{\prime 2} / 2}{a^{2} V+\phi^{\prime 2} / 2}=\frac{m_{p}^{2}}{2}\left(\frac{\partial_{\phi} V}{V}\right)^{2}, \\
\delta & \equiv \frac{\mathcal{H} \phi^{\prime}-\phi^{\prime \prime}}{\mathcal{H} \phi^{2}}=m_{p}^{2} \frac{\partial_{\phi}^{2} V}{V}-\frac{m_{p}^{2}}{2}\left(\frac{\partial_{\phi} V}{V}\right)^{2},
\end{aligned}
$$

with primes denoting derivatives with respect to conformal time. The conditions $\epsilon, \delta \ll 1$ imply that the universe is inflating, with $\epsilon=\delta=0$ corresponding to pure de Sitter expansion. In general, $\epsilon$ and $\delta$ can be functions of time, but in the slow-roll approximation we can take them to be constants.

Perturbing the metric

$$
g_{\mu \nu} d x^{\mu} d x^{\nu} \rightarrow\left(g_{\mu \nu}+\delta g_{\mu \nu}\right) d x^{\mu} d x^{\nu} \equiv a^{2}(\eta)\left[-(1+2 \Phi) d \eta^{2}+(1-2 \Phi) \delta_{i j} d x^{i} d x^{j}\right]
$$

and the scalar field

$$
\phi \rightarrow \phi+\delta \phi
$$

we compute the action for the perturbations to be [26]

$$
S_{\text {pert. }}=\frac{1}{2} \int d^{4} x\left[v^{\prime 2}-(\partial v)^{2}+\frac{z^{\prime \prime}}{z} v^{2}\right] .
$$

Here, $z \equiv \frac{a \phi^{\prime}}{\left(a^{\prime} / a\right)}$, and $\partial$ is the ordinary partial derivative over the three spatial indices. The dynamical degree of freedom of the perturbations, $v \equiv a\left(\delta \phi+\left(\frac{\phi^{\prime}}{a^{\prime} / a}\right) \Phi\right)$, is a linear combination of perturbations in the metric and the scalar field. 
Varying the action (7), we obtain the equation of motion

$$
v^{\prime \prime}+\left(-\partial^{2}-\frac{z^{\prime \prime}}{z}\right) v=0
$$

for the perturbation. We quantize the field $v$ by promoting it to an operator $\hat{v}$ which can be expanded into Fourier modes and their associated raising and lowering operators [26, 27]

$$
\hat{v}=\frac{1}{\sqrt{2}} \int \frac{d^{3} k}{(2 \pi)^{3 / 2}}\left(v_{k}(\eta) e^{-i \mathbf{k} \cdot \mathbf{x}} \hat{a}_{\mathbf{k}}+v_{k}^{*}(\eta) e^{i \mathbf{k} \cdot \mathbf{x}} \hat{a}_{\mathbf{k}}^{\dagger}\right)
$$

The raising and lowering operators obey the commutation relation $\left[\hat{a}_{\mathbf{k}}, \hat{a}_{\mathbf{k}^{\prime}}^{\dagger}\right]=\delta\left(\mathbf{k}-\mathbf{k}^{\prime}\right)$ if the mode functions satisfy the following normalization condition

$$
v_{k}^{\prime} v_{k}^{*}-v_{k}^{\prime *} v_{k}=-2 i
$$

Substituting Eq. (9) into the equation of motion (8), we obtain the the equation of motion for the mode function

$$
v_{k}^{\prime \prime}+\left(k^{2}-\frac{z^{\prime \prime}}{z}\right) v_{k}=0
$$

The quantum field theoretic description is incomplete without a specification for the choice of a vacuum state, defined as a state $|0\rangle$ annihilated by the lowering operator $\left(\hat{a}_{\mathbf{k}}|0\rangle=0\right)$. The choice of a vacuum state $|0\rangle$ of the field is equivalent to choosing the initial conditions for the mode functions $v_{k}$ at some time $\eta_{0}$ [27]. In Minkowski spacetime, the vacuum choice corresponding to the initial conditions

$$
v_{k}\left(\eta_{0}\right)=\frac{1}{\sqrt{k}}, v_{k}^{\prime}\left(\eta_{0}\right)=-i \sqrt{k}
$$

is the unique choice that is invariant under the symmetries of Minkowski spacetime. In curved spacetimes, however, no unique choice exists in general. However, the success of quantum field theory in predicting results of accelerator experiments implies that length scales much smaller than the curvature of spacetime, the vacuum is essentially the same as the Minkowski vacuum. We therefore choose a parametrized set of vacua that reduce to the Minkowski vacuum when the curvature $H^{-1}$ is small compared to the physical length of the mode $k$.

Following Ref. 21], we impose the initial conditions

$$
\begin{aligned}
& v_{k}\left(\eta_{0}\right)=\frac{1}{\sqrt{k}}\left[1+\frac{X+Y}{2} \theta_{0}+\mathcal{O}\left(\theta_{0}^{2}\right)\right], \\
& v_{k}^{\prime}\left(\eta_{0}\right)=-i \sqrt{k}\left[1+\frac{Y-X}{2} \theta_{0}+\mathcal{O}\left(\theta_{0}^{2}\right)\right] .
\end{aligned}
$$

Here, $X$ and $Y$ are two complex parameters with the normalization condition (10) constraining $\operatorname{Re}(Y)$ to be zero. The choice $X=Y=0$ corresponds to the initial conditions for the conventional vacuum choice.

The parameter $\theta_{0}$ is defined as

$$
\left.\theta_{0} \equiv \frac{a H}{k}\right|_{\eta_{0}}
$$

which is the ratio of the physical size of a comoving mode $k$ to the Hubble length at time $\eta_{0}$. Note that $\theta_{0}$ vanishes in the limit when the physical size of the mode $k, \lambda=a / k$, is much smaller than the Hubble radius $\left(\lambda \ll H^{-1}\right)$, as well as when there is no expansion $(H \rightarrow 0)$. In these cases, the comoving mode $k$ does not see the curvature of the Universe, and we recover the Minkowski vacuum as a result.

For each mode, there is an ambiguity in the time $\eta_{0}$ when $v_{k}\left(\eta_{0}\right)$ an $v_{k}^{\prime}\left(\eta_{0}\right)$ are specified. Conventionally, the initial conditions for all of the modes are specified at $\eta_{0} \rightarrow-\infty$. However, that limit corresponds to a vacuum choice made when the physical length of the mode $(k / a)^{-1} \rightarrow 0$, where we expect conventional physics to break down. Following Refs. [19, 20, 28], it seems more sensible to define the vacuum for each mode at the time when the physical length of the mode equals a fixed length scale $\Lambda^{-1}$, so that

$$
\frac{a\left(\eta_{0}(k)\right)}{k}=\Lambda^{-1}
$$


This introduces a cutoff scale $\Lambda$ into the theory, which can be interpreted as the energy scale where the quantum field theoretic description of the perturbations breaks down. The cutoff scale is a free parameter of the theory, although this is normally taken to be the Planck scale. With this prescription, the vacuum for each mode $k$ is defined at a different time $\eta_{0}(k)$, and $\theta_{0}$ has the following scaling relation:

$$
\theta_{0}=\frac{H_{*}}{\Lambda}\left(\frac{k}{k_{*}}\right)^{-\epsilon}
$$

where $H_{*}$ is the Hubble parameter evaluated at the time the physical size of a comoving reference scale $k_{*}$ crosses the cutoff $\Lambda^{-1}$.

In Eq. (13), $X$ and $Y$ can generally be functions of $k$. However, since they are dimensionless quantities, they can only depend on ratios of dimensionful quantities. Without introducing new scales into the theory, the only such ratios are $k / a H$ and higher time derivatives such as $(k / a)^{\prime} / H^{\prime}$. In the former case, we can expand $X$ and $Y$ as functions of $\theta_{0}$ and recover the form of Eqs. (13). In the latter case, we expand $X$ and $Y$ as functions of $\theta_{0}$, the slow-roll parameters, and their time derivatives. Since we have fixed the slow-roll parameters as constants of the theory, they can similarly be absorbed into the parameterization without any loss of generality.

The solution to the equation of motion (11) is given by

$$
v_{k}(\eta)=|\eta|^{1 / 2}\left[A_{k} H_{\nu}^{(1)}(|k \eta|)+B_{k} H_{\nu}^{(1) *}(|k \eta|)\right]
$$

where the Hankel function index is related to the slow-roll parameters by

$$
\nu=\frac{3}{2}+2 \epsilon-\delta
$$

The integration constants $A_{k}$ and $B_{k}$ are fixed by the initial conditions (13) to be

$$
\begin{aligned}
A_{k} & =\sqrt{\frac{\pi}{2}}\left[1+Y \frac{\theta_{0}}{2}-i\left(\frac{2 \nu+1}{4}\right) \theta_{0}\right] e^{i \sigma}, \\
B_{k} & =\sqrt{\frac{\pi}{2}} X \frac{\theta_{0}}{2} e^{i \sigma} \\
\sigma & =\frac{-1}{\theta_{0}(1-\epsilon)}+\pi\left(\frac{\nu}{2}+\frac{1}{4}\right) .
\end{aligned}
$$

Different choices of vacua leave different imprints upon the temperature anisotropies in the cosmic microwave background radiation. For scalar perturbations, the amplitude of the fluctuations can be characterized by the power spectrum of the Bardeen variable $\hat{\zeta} \equiv \hat{v} / z$, defined through the two-point correlation function as

$$
\langle 0|\hat{\zeta}(\mathbf{x}, \eta) \hat{\zeta}(\mathbf{x}+\mathbf{r}, \eta)| 0\rangle \equiv \int \frac{d k}{k} \frac{\sin k r}{k r} \Delta_{\zeta}^{2}(k)
$$

Substituting Eq. (9) into Eq. (22) and using Eq. (3), we find that

$$
\Delta_{\zeta}^{2}(k)=\frac{1}{8 m_{p}^{2} \pi^{2}} \frac{k^{3}}{a^{2} \epsilon}\left|v_{k}\right|^{2} .
$$

With these equations, we can compute the power spectrum for long wavelength modes $|k \eta| \ll 1$, which is the spectrum of fluctuations that seed the microwave background. Substituting Eqs. (17), (19), (20) and $n_{s}=1-4 \epsilon+2 \delta$ into Eq. (23), we obtain the result

$$
\Delta_{\zeta}^{2}(k)=\Delta_{0}^{2}\left\{1+\theta_{0}|X| \cos \left[\frac{2}{\theta_{0}(1-\epsilon)}+\varphi-2 \pi\left(\frac{3-n_{s}}{4}\right)\right]\right\} .
$$

Here, $\varphi$ is the phase of $X$, i.e., $X \equiv|X| e^{i \varphi}$, and $\Delta_{0}^{2}$ is the uncorrected power spectrum

$$
\Delta_{0}^{2}=\delta_{\zeta}^{2}\left(\frac{k}{k_{*}}\right)^{n_{s}-1}
$$

with $\delta_{\zeta}^{2}$ specifying the normalization of the power spectrum. Note that $Y$ does not appear to first order. 
The modification to the power spectrum (24) consists of an oscillatory piece with a $k$-dependent amplitude. A more convenient choice of parameters would be related directly to the characteristics of the oscillation, rather than to the theoretical model. To rewrite the power spectrum, we introduce the following parameters:

$$
\begin{aligned}
\lambda & \equiv|X| \frac{H_{*}}{\Lambda}, \\
\omega & \equiv \frac{2 \epsilon}{1-\epsilon} \frac{\Lambda}{H_{*}} \\
\alpha & \equiv \varphi-2 \pi\left(\frac{3-n_{s}}{4}\right) .
\end{aligned}
$$

The parameter $\lambda$ is the amplitude of the oscillation, modulo the $k$ dependence $\left(k / k_{*}\right)^{-\epsilon}$, and $\omega$ is the oscillation frequency in $\log \left(k / k_{*}\right)$. Lastly, $\alpha$ characterizes the phase of the oscillation. Previous studies [22, 23, 24] do not explicitly include the phase as a free parameter in their computation of constraints. However, since the phase shifts the starting point of the oscillations, this should not be neglected in assessing the observability of the oscillations.

With these parameters, the power spectrum (24) is rewritten in its final form

$$
\Delta_{\zeta}^{2}(k)=\Delta_{0}^{2}\left\{1+\lambda\left(\frac{k}{k_{*}}\right)^{-\epsilon} \cos \left[\frac{\omega}{\epsilon}\left(\frac{k}{k_{*}}\right)^{\epsilon}+\alpha\right]\right\} .
$$

The choice $\lambda=\zeta, \omega=2 \epsilon / \zeta$, and $\alpha=-\pi / 2$ corresponds to the two-parameter model considered in [22], with $\zeta, \epsilon \in[0,1]$.

The amplitude of the oscillation, $|X| \frac{H_{*}}{\Lambda}$, is dependent on the parameters $|X|$ and $\Lambda$, but the frequency $\frac{2 \epsilon}{1-\epsilon} \frac{\Lambda}{H_{*}}$ depends only on $\Lambda$. Thus a measurement of the frequency $\omega$ allows us to break the degeneracy in the amplitude.

Finally we comment on the possible range of values that $|X|$ and $|Y|$ can take. Because Eqs. (13) are expansions in $\theta_{0}$, there is no upper bound for $|X|$ and $|Y|$ if we do not require the expansion to converge. However, there are a couple of reasons why we should expect $|X|$ and $|Y|$ to be small. The first is a theoretical prejudice: if $|X|$ and $|Y|$ are not small, then there is no reason to expect the $\mathcal{O}\left(\theta_{0}^{2}\right)$ and higher-order terms to be negligible. In addition, limits on gravitational particle production may constrain $|X|<5.5 \frac{m_{p}}{\Lambda}[21]$.

\section{B. Primordial Power Spectrum to the CMB}

The temperature and polarization anisotropies of the microwave background arise from acoustic oscillations of the inhomogeneous photon-baryon fluid, which are seeded by the metric fluctuations created during inflation. The multipole moments of the fluctuations can be related to the Bardeen variable $\zeta(\mathbf{k})$ as

$$
X_{l}^{m}=\frac{4 \pi}{\sqrt{2 l(l+1)}}(-i)^{l} \int \frac{d^{3} k}{(2 \pi)^{3}} T_{l}^{X}(k) Y_{l}^{m *}(\hat{\mathbf{k}}) \zeta(\mathbf{k})
$$

where $X=\{T, E\}$ is the temperature or $E$-mode multipole moments, and $T_{l}^{X}(k)$ denotes the transfer function encoding the acoustic physics and the projection effects (see, e.g., Ref. [15] for discussion on the structure of the transfer functions).

The CMB power spectra, defined as

$$
\left\langle X_{l}^{m *} X_{l^{\prime}}^{\prime m^{\prime}}\right\rangle \equiv C_{l}^{X X^{\prime}} \delta_{l l^{\prime}} \delta_{m m^{\prime}}
$$

are then related to the primordial power spectrum $\Delta_{\zeta}^{2}(k)$ by

$$
\frac{l(l+1)}{2 \pi} C_{l}^{X X^{\prime}}=\int \frac{d k}{k} T_{l}^{X}(k) T_{l}^{X^{\prime}}(k) \Delta_{\zeta}^{2}(k) .
$$

The transfer functions in Eq. (32) limit our ability to measure $\Delta_{\zeta}^{2}(k)$ in several ways. First, $T_{l}^{X}(k)$ projects the fluctuations onto a sphere, and this projection peaks along $l_{\text {peak }} \approx k r_{*}$ with a width of $\Delta l \approx 20$, where $r_{*}=13.8 \mathrm{Gpc}$ is the angular diameter distance to the last scattering surface. Since a Fourier mode with wave number $k$ projected onto a sphere also contributes to multipole moments with $l \ll l_{\text {peak }}$, power at wave number $k$ gets distributed to multipole moments with $l \ll l_{\text {peak }}$, as well as in the narrow band with $\Delta l \approx 20$ around $l \ll l_{\text {peak }}$. 
The transfer functions $T_{k}^{X}(k)$ also include contributions that arise due to secondary effects. In the low multipole regime, the temperature transfer function includes a contribution from the integrated Sachs-Wolfe effect [29], which provides additional power at lower multipoles. Rescattering of the CMB photons during reionization contributes to a feature in the low multipole regime of the polarization transfer function. This shows up as a bump in the EE power spectrum and $T E$ cross spectrum, of which the latter was first detected by the WMAP collaboration [30]. Both features tends to broaden features in the primordial power spectrum. Although there are other foreground effects at high multipoles, we work in the multipole regime below which those secondaries are insignificant.

An additional limitation arises due to gravitational lensing of the CMB [31, 32, 33]. Lensing has the effect of redistributing power between adjacent multipole bins, thereby reducing the width of resolvable features to roughly $\Delta l \sim 60$. This translates to a maximum log frequency of roughly $\omega \lesssim 30$, and potentially an important limitation on measuring the properties of the primordial fluctuations [15]. We will restrict the range of $\omega$ to be below 30 in our analysis to avoid the regime where contamination due to lensing is an important effect.

\section{ANALYSIS}

We are interested in how well the microwave background can in principle distinguish signatures of new physics from the standard inflationary models. Previous works have estimated the uncertainties on parameters related to the cutoff physics using the Fisher matrix formalism [24], or used a likelihood approach on a fiducial model containing a modulation 22]. Both start from a two-parameter model for the primordial power spectrum [19], and therefore do not incorporate the full range of effects that might arise from the existence of a high-energy cutoff. Furthermore, the analyses of the ideal case depend on the choice of fiducial models. We currently have no a priori knowledge, either from experiments or theoretical prejudice, about what set of parameters would constitute a reasonable fiducial model other than the standard inflationary model without oscillations. However, Refs. 24] and [22] both assume fiducial models containing oscillations, and the resulting parameter forecasts therefore only apply to those fiducial models.

In this work, we instead present two analyses, both of which complement the existing literature. In the first, we use current $\mathrm{CMB}$ data sets to place constraints on the four-parameter description of the oscillation induced by the phenomenology at the cutoff scale. In the second, we present the fundamental limitations on measurements of the oscillation parameters that arise from the statistics of CMB measurements.

Both of these analyses rely on the calculation of the likelihood over the expanded parameter set, which is accomplished using Markov Chain Monte Carlo (MCMC) methods. In the following sections, we briefly describe the calculation of likelihoods for microwave background data, as well as details concerning our use of MCMC.

\section{A. Likelihood Analysis}

Let us suppose that we are given a data set $\hat{C}_{l}$, along with a set of model power spectra $C_{l}(\mathbf{p})$, with $\mathbf{p}$ denoting a vector in an $N$-dimensional parameter space. The posterior probability distribution $P\left(\mathbf{p} \mid \hat{C}_{l}\right)$, which is the probability density at point $\mathbf{p}$ in the parameter space given the data set, is then computed by

$$
P\left(\mathbf{p} \mid \hat{C}_{l}\right) \propto P(\mathbf{p}) \mathcal{L}\left(\hat{C}_{l} \mid \mathbf{p}\right) .
$$

Here, $P(\mathbf{p})$ represents our prior assumptions about the parameters, and $\mathcal{L}$ denotes the likelihood function. The proportionality constant is set by requiring that

$$
\int d \mathbf{p} P\left(\mathbf{p} \mid \hat{C}_{l}\right)=1
$$

over the allowed range in parameter space. In many instances, we do not have any prior knowledge about what the parameter should be, so that we assign a flat prior $P\left(p^{i}\right)=1$ for the $i$ th parameter. In such a case, the posterior probability is proportional to the likelihood function, so maximizing the likelihood function in the parameters is equivalent to finding the point with the maximum probability density. To find the $1 \sigma$ uncertainties around the maximum likelihood point, we then find contours that enclose $68.3 \%$ of the posterior probability.

Assuming that the CMB multipole moments are Gaussian distributed, the likelihood for an all-sky, noiseless experiment is given by

$$
\mathcal{L} \propto \prod_{l m} \frac{1}{\sqrt{\operatorname{det} \mathbf{C}_{l}}} \exp \left\{-\frac{1}{2} \mathbf{d}_{l}^{\dagger m} \mathbf{C}_{l}^{-1} \mathbf{d}_{l}^{m}\right\}
$$


where the data vector is given by $\mathbf{d}_{l}^{m} \equiv\left\{T_{l}^{m}, E_{l}^{m}\right\}$, and the covariance matrix is

$$
\mathbf{C}_{l}=\left(\begin{array}{cc}
C_{l}^{T T} & C_{l}^{T E} \\
C_{l}^{T E} & C_{l}^{E E}
\end{array}\right)
$$

with $C_{l}^{X X^{\prime}}$ denoting the theoretical power spectra. Defining the estimator for the power spectra according to

$$
\hat{C}_{l}^{X X^{\prime}} \equiv \sum_{m} \frac{\left|X_{l}^{* m} X_{l}^{\prime m}\right|}{2 l+1}
$$

the log likelihood function becomes (up to an irrelevant constant)

$$
-2 \ln \mathcal{L}=\sum_{l}(2 l+1)\left[\ln \left(\frac{C_{l}^{T T} C_{l}^{E E}-\left(C_{l}^{T E}\right)^{2}}{\hat{C}_{l}^{T T} \hat{C}_{l}^{E E}-\left(\hat{C}_{l}^{T E}\right)^{2}}\right)+\frac{\hat{C}_{l}^{T T} C_{l}^{E E}+\hat{C}_{l}^{E E} C_{l}^{T T}-2 \hat{C}_{l}^{T E} C_{l}^{T E}}{C_{l}^{T T} C_{l}^{E E}-\left(C_{l}^{T E}\right)^{2}}-2\right]
$$

We have chosen the constants so that $-2 \ln \mathcal{L}=0$ when the theoretical power spectra $C_{l}^{X X^{\prime}}$ are equal to the data estimates $\hat{C}_{l}^{X X^{\prime}}$, such that $-2 \ln \mathcal{L}$ behaves as an effective chi-squared statistic. We will use this form of the likelihood function in our analysis of the idealized case.

If only the temperature power spectrum is measured, then the log likelihood function is given by

$$
-2 \ln \mathcal{L}^{T T}=\sum_{l}(2 l+1)\left[\ln \left(\frac{C_{l}^{T T}}{\hat{C}_{l}^{T T}}\right)+\frac{\hat{C}_{l}^{T T}}{C_{l}^{T T}}-1\right]
$$

For a realistic experiment, the presence of sky cuts and instrumental noise necessitate the use of various approximations to the likelihoods, Eqs. (38) and (39).

The transfer functions $T_{l}^{X}(k)$ used to calculate the model power spectra $C_{l}^{X X^{\prime}}$ can be obtained by using publicly available Boltzmann integral codes such as CMBFAST [34] and CAMB [35]. However, these codes sample the transfer function sparsely in $k$ and $l$ to reduce computation time, and the resulting power spectra can deviate from the true power spectra if there are features in the initial power spectrum which have scales comparable to the gridding. In our parametrization, higher values of $\omega$ correspond to higher oscillation frequencies, so we expect the computation from fast Boltzmann codes to become less reliable in that regime.

To illustrate this, we compare the power spectra generated using CAMB to those from a Boltzmann hierarchy code developed by $\mathrm{Hu}$ [15], which has the advantage of capturing the high frequency oscillations that might be problematic with the integral codes. To determine the frequency limit imposed by the accuracy of the code, we compare the power spectra generated by CAMB to that from the hierarchy code for various values of $\omega$. As can be seen in Fig. 1 where we plot the fractional deviation

$$
\frac{\Delta C_{l}}{C_{l}} \equiv \frac{C_{l}^{\mathrm{CAMB}}-C_{l}^{\mathrm{true}}}{C_{l}^{\mathrm{true}}}
$$

the amplitude of the deviation increases with $\omega$. We impose the fairly strict condition that deviations be less than $0.2 \%$. As a result, the range of $\omega$ is restricted to be less than 20 for computations performed using CAMB.

Since computing the likelihood function from $T_{l}^{X}(k)$ on a grid over the entire parameter space is generally not feasible for more than two parameters, we utilize Markov Chain Monte Carlo sampling to compute the posterior probability distribution.

\section{B. Markov Chain Monte Carlo}

Markov Chain Monte Carlo (MCMC) methods have been applied to CMB analysis of the data from several experiments [36, 37]. We give a brief overview of the method, as well as a description of our implementation, and refer the reader to Refs. 36, 38] for full treatments.

MCMC is a method designed to simulate the posterior probability distribution through a series of random draws. In our case, the MCMC produces a set of $N$ points $\left\{\mathbf{p}_{t}, t=1 \ldots N\right\}$ that fairly sample the distribution $P\left(\mathbf{p} \mid \hat{C}_{l}\right)$, so that the density of points $\left\{\mathbf{p}_{t}\right\}$ is proportional to the posterior distribution. Statistical properties of the parameters 
$p^{i}$ can then be estimated using the corresponding properties of the samples; for instance, the mean and variance of parameter $p^{i}$ can be estimated by using the sample mean and variance via

$$
\begin{aligned}
\bar{p}^{i} & =\frac{1}{N} \sum_{t=1}^{N} p_{t}^{i} \\
\sigma^{2}\left(p^{i}\right) & =\frac{1}{N-1} \sum_{t=1}^{N}\left(p_{t}^{i}-\bar{p}^{i}\right)^{2} .
\end{aligned}
$$

Our goal is to produce a chain whose stationary distribution is the distribution $P\left(\mathbf{p} \mid \hat{C}_{l}\right)$. We describe the MetropolisHastings algorithm [39, 40], used in many MCMC implementations for CMB analysis including the WMAP analysis [37] and the public package CosmoMC [36], as well as in our own implementation. We refer the reader to Ref. 36] for details on CosmoMC, which we utilize in Sec. IV and give a generic overview of the algorithm here.

At each step $t$ in this algorithm, a candidate point $\mathbf{q}$ is first chosen from a proposal distribution $\beta\left(\mathbf{q} \mid \mathbf{p}_{t}\right)$. The point $\mathbf{q}$ is accepted with probability

$$
\pi\left(\mathbf{p}_{t}, \mathbf{q}\right)=\min \left(1, \frac{P\left(\mathbf{q} \mid \hat{C}_{l}\right) \beta\left(\mathbf{p}_{t} \mid \mathbf{q}\right)}{P\left(\mathbf{p}_{t} \mid \hat{C}_{l}\right) \beta\left(\mathbf{q} \mid \mathbf{p}_{t}\right)}\right)
$$

If $\mathbf{q}$ is accepted, then the chain continues, with $\mathbf{p}_{t+1}=\mathbf{q}$; otherwise, $\mathbf{p}_{t+1}=\mathbf{p}_{t}$, i.e., the chain does not move. Note that the proposal distribution can depend on the last point of the chain. We take $\beta\left(\mathbf{q} \mid \mathbf{p}_{t}\right)$ to be a Gaussian distribution with mean $\mathbf{p}_{t}$ and a fixed, diagonal covariance matrix. We first run a short Markov chain to determine the variance to be used in the proposal distribution.

Proper conclusions from chains are not possible without determining if the chains have converged to the stationary distribution. The first step in ensuring this is to remove the first few thousand points belonging to a burn-in period. All points past the burn-in period are assumed to have forgotten about its starting point, and can be thought of as being sampled from the stationary distribution.

Additionally, we employ a convergence criterion, advocated by Gelman and Rubin 41] and used by the WMAP collaboration [2, 3, 37], to ensure proper mixing and convergence of chains. In this criterion, one generates several chains from different starting points, and quantifies whether these chains are indistinguishable from each other.

Suppose one generates $M$ chains from different starting points, each chain with $2 N$ points. For each parameter $p^{i}$, we take the last $N$ points from each chain, and label the sequences as $\left\{p_{\alpha \beta}^{i}\right\}$, with $\alpha=1, \ldots, N$, and $\beta=1, \ldots, M$. Let the mean of each chain be defined by

$$
\bar{p}_{\beta}^{i}=\frac{1}{N} \sum_{\alpha=1}^{N} p_{\alpha \beta}^{i}
$$

and the mean of the distribution by

$$
\bar{p}^{i}=\frac{1}{M N} \sum_{\alpha=1}^{N} \sum_{\beta=1}^{M} p_{\alpha \beta}^{i} .
$$

We then define the variance between chains $B^{i}$ and the variance within chains $W^{i}$ as

$$
\begin{aligned}
B^{i} & =\frac{N}{M-1} \sum_{\beta=1}^{M}\left(\bar{p}_{\beta}^{i}-\bar{p}^{i}\right)^{2}, \\
W^{i} & =\frac{1}{M(N-1)} \sum_{\beta=1}^{M} \sum_{\alpha=1}^{N}\left(p_{\alpha \beta}^{i}-\bar{p}_{\beta}^{i}\right)^{2} .
\end{aligned}
$$

Lastly, we can define an estimate of the variance of $p^{i}$ using

$$
V^{i}=\frac{N-1}{N} W^{i}+\frac{1}{N} B^{i}
$$

The estimate $V^{i}$ is an unbiased estimator of the variance if the chains are stationary, but overestimates the variance if the starting points of the chains are dispersed. On the other hand, $W^{i}$ typically underestimates the variance, since 
the finite chains would not have had enough steps to range over the entire distribution. Both $V^{i}$ and $W^{i}$ approach the true variance in the limit $N \rightarrow \infty$, but from opposite sides. If we now form the ratio

$$
R^{i} \equiv \frac{V^{i}}{W^{i}},
$$

this ratio should approach 1 from above as the chains grow long, i.e., as the chains more faithfully reproduces the target distribution, and small values of $R^{i}$ indicate that the chains have mixed well and converged. We take the condition $R^{i}<1.1$ for all parameters as our convergence criterion.

\section{CURRENT BOUNDS FROM CMB DATA}

We first present the constraints on the oscillation parameters using the WMAPext data set [2], which consists of the WMAP 1-year data set 30] supplemented by the Arcminute Bolometer Array Receiver(ACBAR) [42] and Cosmic Background Imager (CBI) [43] data sets to extend the multipole range. Although this analysis is similar to those presented in [22, 23, 24], our parametrization of the primordial power spectrum removes some of the degeneracies present in the original parametrization.

In our analysis, we focus on the constraints that can be placed on the parameters defining the oscillations in the presence of cosmological parameters. We vary the following four cosmological parameters $\left(\Omega_{b} h^{2}, \Omega_{\mathrm{CDM}} h^{2}, H_{0}, z_{\mathrm{re}}\right)$, as well as the six parameters $\left(A_{s}, n_{s}, \lambda, \epsilon, \omega, \alpha\right)$ that define the primordial power spectrum, assuming flatness of the universe. Here, $A_{s}$ parametrizes the normalization of the primordial power spectrum according to

$$
\delta_{\zeta}^{2}=10^{-10} A_{s},
$$

and matches the normalization convention used in CAMB.

The likelihood contours are calculated using CosmoMC, with the power spectra computed from a version of CAMB modified to incorporate the oscillatory power spectrum. We assume a flat prior for all parameters, restricting the ranges of $\omega$ and $\epsilon$ to be $\omega<20$ and $\epsilon<0.2$, respectively.

Our results are based on eight chains started at random points in the 10-dimensional parameter space. We find that the chains have converged after 100000 points, using the convergence criterion presented in Sec. IIIB

The limits on the oscillation parameters are given in Table I with the corresponding likelihood contours shown in Fig. 2 Not surprisingly, the current data set does not give a strong constraint. At $2 \sigma$, the amplitude of the oscillations are limited to $\lambda<0.77$, indicating consistency with a scale-invariant power spectrum. Assuming $|X|=1$, this translates to a restriction of the cutoff scale to $\Lambda>1.3 H_{\mathrm{infl}}$, and does not lead to any conclusions if we allow $|X|<1$.

The lack of a strong constraint on $\lambda$ is largely due to a large degeneracy between three parameters for a nonoscillatory fiducial model. Specifically, the three subspaces with $\lambda \rightarrow 0, \omega \rightarrow 0$, and $\epsilon \rightarrow 0$ all correspond to lack of oscillations, so that a data set consistent with a lack of oscillations will have flat likelihoods along these planes. This behavior can be seen in the likelihood contours of Fig. 3. where we have marginalized the likelihood over all parameters except $\lambda$ and $\omega$. As can be seen, the contours curve along the $\lambda=0$ and $\omega=0$ lines, indicating that both $\lambda=0$ and $\omega=0$ are supported, so that strong constraints cannot be placed on either of the two parameters.

Previous constraints using the current data set come from Refs. [22, 23]. Reference [22] finds that the current data set is consistent with a scale-invariant power spectrum, only excluding the the region with $\lambda>0.1$ and $0.1 \leq \epsilon \leq 1$. Since our results indicate that, for the $\lambda-\epsilon$ plane, the exclusion region is given by $\lambda>0.5, \epsilon>0.05$ to $2 \sigma$, the two analyses are roughly in agreement.

In contrast, Ref. 23] finds that a model with $(\lambda=0.29, \omega=288, \epsilon=0.02)$ has an effective $\chi^{2}$ that is lower than the standard model without oscillations, with a greater than $3 \sigma$ significance. We deliberately avoid the high-frequency regime, since gravitational lensing tends to suppress the oscillatory features for models with $\omega>30$. We therefore cannot make any statements regarding the consistency of the results 47 .

Lastly, we comment on the relation between the cosmological parameters and the oscillation parameters. As expected, we find that the cosmological parameters are essentially independent from the oscillation parameters. The cosmological parameters (e.g., $\Omega_{b} h^{2}$ ) influence the structure of the acoustic peaks, which have roughly equal spacings in $k$. On the other hand, the oscillations we consider are in $\log \left(k / k_{*}\right)$, and intrinsically vary separately from the acoustic peaks. As an illustration, we show the likelihood contours for $\Omega_{\mathrm{CDM}} h^{2}$ and $\epsilon$ in Fig. [4 Thus, the additional parameters do not bias the measurements of the cosmological parameters. 


\section{THEORETICAL LIMIT}

We are ultimately interested in the intrinsic limitations of the CMB as a probe of primordial physics. To investigate this, we now consider an experiment that is noiseless and measures the multipole moments out to some maximum multipole $l_{\max }$, so that the uncertainties in the measurement are due to cosmic variance alone. With this idealized experiment, we use the transfer functions from the hierarchy code and explore the six-dimensional parameter space defining the primordial power spectrum with high precision. Due to the computational expense required, we choose to keep the cosmological parameters fixed. While such a situation may be unrealistic, the resulting limit on our ability to measure the parameters of the primordial power spectrum is robust, in that any consideration of realistic effects can only make the uncertainties larger. Furthermore, since the results from the previous section indicate that the cosmological parameters are independent of the oscillatory parameters to $\omega_{\max }=20$, fixing the cosmological parameters should not bias our results. Thus, the limitations on parameters we quote should be considered as theoretical limits on the detectability of cutoff physics, arising purely from the statistical properties of the microwave background and the processing due to the radiation transfer functions.

The maximum multipole $l_{\max }$ of the idealized experiment is set by considering the additional complications due to gravitational lensing and other secondary effects. The power from gravitational lensing becomes comparable to that from the primary anisotropies at $l \approx 3000$, and presents a fundamental limit to the range of multipoles we can use 33 . However, other secondary effects, such as the thermal and kinetic Sunyaev-Z'eldovich effect [44], become dominant by $l \sim 3000$. Furthermore, deep field observations by CBI suggest an excess of power in the range $l \approx 2000-3500$, which may be due to the aforementioned secondary effects [45]. We therefore choose a conservative value of $l_{\text {max }}=2000$ to avoid the regime where secondary effects may be dominant.

For this ideal case, we simulate a data set by generating a realization of CMB power spectra that corresponds to a primordial power spectrum with $\delta_{\zeta}=5.07 \times 10^{-5}, n_{s}=0.99$, and without the oscillatory features. For convenience, we reparametrize the normalization to

$$
\delta_{\zeta}^{2}=\left(5.07 \times 10^{-5}\right)^{2} A_{f}
$$

so that $A_{f}=1$ corresponds to the fiducial model. This is related to the normalization $A_{s}$ by $A_{f}=3.89 \times 10^{-2} A_{s}$, and is chosen for computational convenience. The cosmological parameters are fixed to be $\left\{\Omega_{b} h^{2}=0.024, \Omega_{m} h^{2}=\right.$ $\left.0.1399, \Omega_{D E}=0.73, \tau=0.17, T / S=0, w_{\mathrm{DE}}=-1\right\}$ for both the simulated data set and the model power spectra. From a converged run consisting of three chains with 230000 points per chain, we find the maximum likelihood point and uncertainties on the six parameters $\left\{A_{f}, n_{s}, \lambda, \epsilon, \omega, \alpha\right\}$ that specify the primordial power spectrum, where we impose the priors $\epsilon<0.2$ and $\omega<30$ to avoid the regime where gravitational lensing becomes important. Gravitational waves are assumed to be absent.

Since we have taken the oscillations to be absent in our simulated data set, the resulting constraints on the oscillation parameters can be regarded as the extent to which the oscillatory parameters can deviate from the fiducial model before being statistically different from the standard case. For instance, a constraint of $\lambda<0.1$ at $1 \sigma$ would imply that models with $\lambda<0.1$ are statistically indistinguishable from the standard model with $\lambda=0$. Such a bound can be thought of as the fundamental lower limit achievable by a CMB experiment.

We present the limits in Table II and the likelihood contours in Fig. 5] showing that a cosmic variance limited experiment to $l \approx 2000$ can limit the oscillations to an amplitude of less than $0.5 \%$ and frequencies of $\omega>6.5$, although other parameters are less constrained. As noted in Ref. 22], the likelihood function has many local maxima and irregularities. Our parametrization seems to reduce the irregularities in the amplitude parameter $\lambda$; however, features are evident in the two-dimensional likelihood contours of the other parameters. While we believe our chains have converged, the presence of such features in the contour plots suggest that robust predictions for parameter uncertainties may require calculation of the likelihood function on much finer intervals.

In particular, a plot of the likelihood function over $\epsilon$ and $\omega$, with fixed amplitude and phase, reveals that the local maxima are distributed along lines of constant $\omega / \epsilon$, and become sparse between $\omega=4$ and $\omega=5$ (Fig. 6). The distribution along constant $\omega / \epsilon$ can be understood as follows. Given that the oscillation for $\alpha=0$ is given by

$$
\cos \left[\frac{\omega}{\epsilon}\left(\frac{k}{k_{*}}\right)^{\epsilon}\right],
$$

shifting $\omega$ by $2 n \pi \epsilon /\left(k / k_{*}\right)^{\epsilon}$ for some integer $n$ does not change the value of the cosine at wave number $k$. Furthermore, $\left(k / k_{*}\right)^{\epsilon}$ is a slowly varying function of $\epsilon$, so that the value of the cosine is roughly constant for $\omega / \epsilon$ in integer multiples of $2 \pi /\left(k / k_{*}\right)^{\epsilon}$. If we now make a simplifying assumption that the likelihood is dominated by contributions from a small range in $k$ around some value $k_{d}$, then the likelihood function should exhibit this multiplicity as structures along

$$
\frac{\omega}{\epsilon}=\frac{2 n \pi}{\left(k_{d} / k_{*}\right)^{\epsilon}} .
$$


The low likelihood in the range $4<\omega<5$ can be explained by noting the frequency of oscillatory peaks and troughs in each decade. In each decade, there are approximately $\omega \ln (10) / 2 \pi$ oscillations, so that in the range $4<\omega<5$, this corresponds to roughly three extrema in the oscillatory features (see Fig. 17). The extrema in the oscillations tend to coincide with the $\mathrm{CMB}$ acoustic peaks, and the overall effect of the primordial oscillations is to change the heights of the acoustic peaks. As is well known, the ratio of heights of the CMB acoustic peaks can be constrained tightly, and as a result, the likelihoods for $4<\omega<5$ tend to be lower. Because our MCMC sampler moves the chain based on the ratio of likelihoods, the lack of high likelihood regions between $\omega=4$ and $\omega=5$ implies that the chains do not tend to cross into this region, possibly explaining the fact that our chains do not explore the region below $\omega=5$.

Given the presence of such irregularities, obtaining reliable constraints on the oscillation parameters is difficult. We believe that our reparametrization of the oscillation parameters into physically distinct parameters reduces the irregularities in the amplitude parameter, making the results concerning $\lambda$ robust. However, to obtain robust limits on the other parameters, the presence of multiple maxima needs to be taken into account.

In MCMC analysis, the step sizes are typically taken to be similar to the characteristic size of the (hopefully global) maximum of the likelihood. However, if the local maxima have similar sizes, the chains can get stuck in the local maxima for a long time and lead to false conclusions about the location and constraints from the chains. In exploring these models, it is therefore important to monitor convergence using multiple chains, since it is unlikely that chains started from different, randomly chosen starting points would all fall into the same false local maximum. Furthermore, nonstandard algorithms (e.g., high-temperature chains [46] and other modified samplers [38]) might be necessary to improve mixing of the chains, allowing exploration of the irregular features in a reasonable amount of computation time.

\section{DISCUSSION}

We have performed a full likelihood analysis of the detectability of oscillatory primordial power spectra in current and future CMB data, motivated by a phenomenological description of possible effects on the primordial power spectrum due to the presence of a high-energy cutoff [21]. Not surprisingly, only a weak constraint can be placed on the parameters that define the oscillations, the current data set being consistent with a scale-invariant power spectrum without oscillations.

The analysis of the fundamental limitation from a cosmic variance limited experiment gives us cause for some cautious optimism, suggesting that the amplitude of the oscillations can be limited to less than $0.5 \%$. Under the assumption that the vacuum choice parameter $|X| \approx 1$, this translates to a possible limit on the cutoff energy scale of $\Lambda>200 H_{\text {infl }}$. Stated differently, if $\Lambda<200 H_{\text {infl }}$, the resulting features in the primordial power spectrum is observable in principle, allowing such a model to be distinguished from the null model. Note that this is currently the only limit on the cutoff scale using the effects on the vacuum choice. As noted above, however, the limits on the other parameters may not be robust due to the fact that the likelihood function exhibits multiple local maxima. Future analyses may need to take this into account by utilizing MCMC techniques that facilitate jumps between maxima, such as modifications to the sampling method.

Is it possible to do better? At present, the CMB remains the best tool for exploring the shape of the primordial power spectrum, limited fundamentally by gravitational lensing due to the intervening matter [15]. Other probes of cosmological fluctuations, such as weak lensing surveys, can help extend the wave number range that can be probed, but exploring the effect of uncertainties from such surveys is beyond the scope of this work.

Given the complex structure of the likelihood function, robust results from high-precision surveys will require the use of modified MCMC algorithms in order to map out the multiple features in the likelihood. With that caveat, future surveys, such as the Planck and CMBPol missions, should be able to limit the shape of the primordial power spectrum and provide an insight into the nature of the high energy cutoff.

\section{Acknowledgments}

We acknowledge the use of transfer functions from the hierarchy code written by W. Hu. We thank C. ArmendarizPicon, S. Carroll, W. Hu, J. Martin, D. Nagai, and J. Ruhl for useful comments and discussions. T.O. was supported by NASA NAG5-10840 and the US DOE OJI program. E.A.L. was supported by the US DOE grant DE-FG02- 
90ER40560 and the David and Lucille Packard Foundation.

[1] C. L. Bennett et al., Astrophys. J. Suppl. 148, 1 (2003).

[2] D. N. Spergel et al., Astrophys. J. Suppl. 148, 175 (2003).

[3] H. V. Peiris et al., Astrophys. J. Supp. 148, 213 (2003).

[4] A. H. Guth, Phys. Rev. D 23, 347 (1981).

[5] J. M. Bardeen, P. J. Steinhardt, and M. S. Turner, Phys. Rev. D 28, 679 (1983).

[6] A. A. Starobinsky, Phys. Lett. B 117, 175 (1982).

[7] http://astro.estec.esa.nl/Planck

[8] http://universe.gsfc.nasa.gov/be/roadmap

[9] P. Mukherjee and Y. Wang, Astrophys. J. 593, 38 (2003).

[10] Y. Wang and G. Mathews, Astrophys. J. 573, 1 (2002).

[11] Y. Wang, D. N. Spergel, and M. A. Strauss, Astrophys. J. 510, 20 (1999).

[12] S. L. Bridle, A. M. Lewis, J. Weller, and G. Efstathiou, MNRAS Lett. 342, 72 (2003).

[13] C. J. Miller, R. C. Nichol, C. Genovese, and L. Wasserman, Astrophys. J. 565, 67 (2002).

[14] M. Tegmark and M. Zaldarriaga, Phys. Rev. D 66, 103508 (2002).

[15] W. Hu and T. Okamoto, Phys. Rev. D 69, 043004 (2004).

[16] C. P. Burgess, J. M. Cline, F. Lemieux and R. Holman, JHEP 0302, 048 (2003).

[17] R. H. Brandenberger and J. Martin, Mod. Phys. Lett A 16, 999 (2001).

[18] J. Martin and R. H. Brandenberger, Phys. Rev. D 63, 123501 (2001).

[19] U. H. Danielsson, Phys. Rev. D 66, 023511 (2002).

[20] R. Easther, B. R. Greene, W. H. Kinney and G. Shiu, Phys. Rev. D 66023518 (2002).

[21] C. Armendariz-Picon and E. A. Lim, J. Cosmol. Astropart. Phys. 12, 06 (2003). hep-th/0303103.

[22] Ø. Elgarøy and S. Hannestad, Phys. Rev. D 68, 123513 (2003).

[23] J. Martin and C. Ringeval, Phys. Rev. D (to be published), astro-ph/0310382 (2003).

[24] L. Bergström and U. H. Danielsson, JHEP 0212, 038 (2002).

[25] A. R. Liddle and D. H. Lyth, Cosmological Inflation and Large-Scale Structure (Cambridge University Press, Cambridge, U.K., 2000).

[26] V. F. Mukhanov, H. A. Feldman, and R. H. Brandenberger, Phys. Rep. 215, 203 (1991).

[27] N. D. Birrell and P. C. W. Davies, Quantum Fields in Curved Space (Cambridge University Press, Cambridge, U.K., 1982).

[28] T. Jacobson, Phys. Rev. D D48, 728 (1993).

[29] R. K. Sachs and A. M. Wolfe, Astrophys. J. 147, 73 (1967).

[30] A. Kogut, et al., Astrophys. J. Supp. 148, 161(2003).

[31] U. Seljak, Astrophys. J. 463, 1 (1996).

[32] M. Zaldarriaga and U. Seljak, Phys. Rev. D 58, 023003 (1998).

[33] W. Hu, Phys. Rev. D 62, 043007 (2000).

[34] U. Seljak and M. Zaldarriaga, Astrophys. J. 469, 437 (1996).

[35] A. M. Lewis, A. Challinor, and A. Lasenby, Astrophys. J. 538, 473 (2000).

[36] A. Lewis and S. Bridle, Phys. Rev. D 66103511 (2002).

[37] L. Verde, et al., Astrophys. J. Supp. 148,195 (2003).

[38] W. R. Gilks, S. Richardson, and E. J. Spiegelhalter, ed., Markov Chain Monte Carlo in Practice (Chapman and Hall, London, U.K., 1996).

[39] N. Metropolis et al., J. Chem. Phys. 21, 1087 (1953).

[40] W. K. Hastings, Biometrika 57, 97 (1970).

[41] A. Gelman and D. B. Rubin, Statist. Sci. 7, 457 (1992).

[42] C.-L. Kuo, et al., BAAS 34, 1324 (2002).

[43] T. J. Pearson, et al., Astrophys. J. 591, 556(2003).

[44] R. A. Sunyaev and Y. B. Zel'dovich, Astrophys. Sp. Phys. 4, 173 (1972).

[45] B. S. Mason, et al., Astrophys. J. 591,540 (2003).

[46] C. Jennison, J. R. Statist. Soc. B 55, 54 (1993).

[47] See, however, astro-ph/0402609. 


\section{Tables}

\begin{tabular}{l|cc} 
Parameter & $1 \sigma$ & $2 \sigma$ \\
\hline$\lambda$ & $<0.20$ & $<0.77$ \\
$\epsilon$ & - & - \\
$\omega$ & $<10.1$ & $<17.8$ \\
$\alpha$ & - & -
\end{tabular}

TABLE I: Constraints on $\Delta_{\zeta}^{2}(k)$ parameters from the WMAPext data set, indicating the $1 \sigma$ and $2 \sigma$ uncertainties on parameters. Dashes indicate that no meaningful constraints were possible.

\begin{tabular}{l|ccc} 
Parameter & mean & $1 \sigma$ & $2 \sigma$ \\
\hline$\lambda$ & 0.002 & $\lambda<0.003$ & $\lambda<0.005$ \\
$\epsilon$ & 0.10 & $0.07<\epsilon<0.14$ & $0.002<\epsilon<0.19$ \\
$\omega$ & 18.5 & $16.1<\omega<22.3$ & $6.8<\omega<28.6$ \\
$\alpha$ & 3.6 & $2.6<\alpha<4.7$ & $0.7<\alpha<6.0$
\end{tabular}

TABLE II: Constraints on $\Delta_{\zeta}^{2}(k)$ parameters for a cosmic variance limited experiment, assuming a fiducial model without oscillations. In particular, the amplitude of oscillations can be constrained to be below $0.5 \%$.

Figures

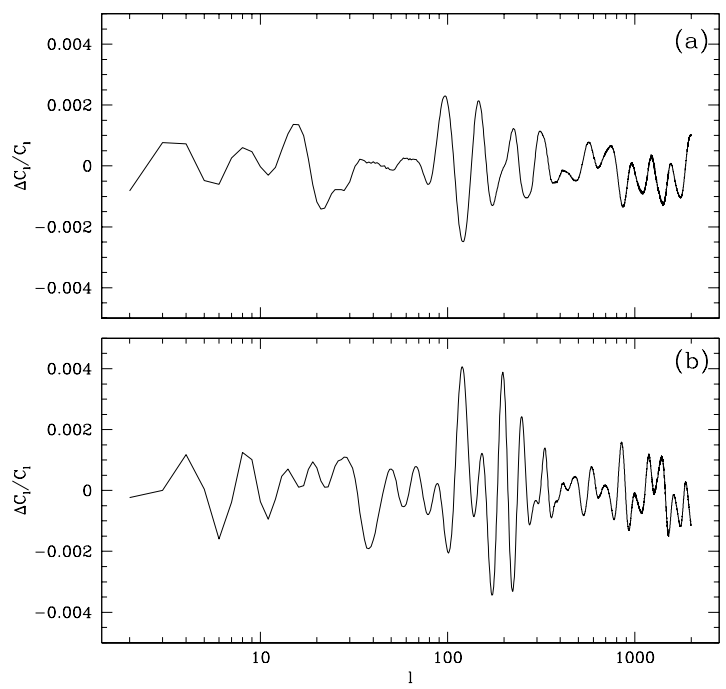

FIG. 1: Fractional deviation of CAMB computation from the true power spectrum, for (a) $\lambda=0.01, \epsilon=0.2, \omega=20, \alpha=0$, and (b) $\lambda=0.01, \epsilon=0.2, \omega=30, \alpha=0$. At $\omega=20$, the deviations are at around $0.2 \%$, while at $\omega=30$, the deviations approach $0.4 \%$. 

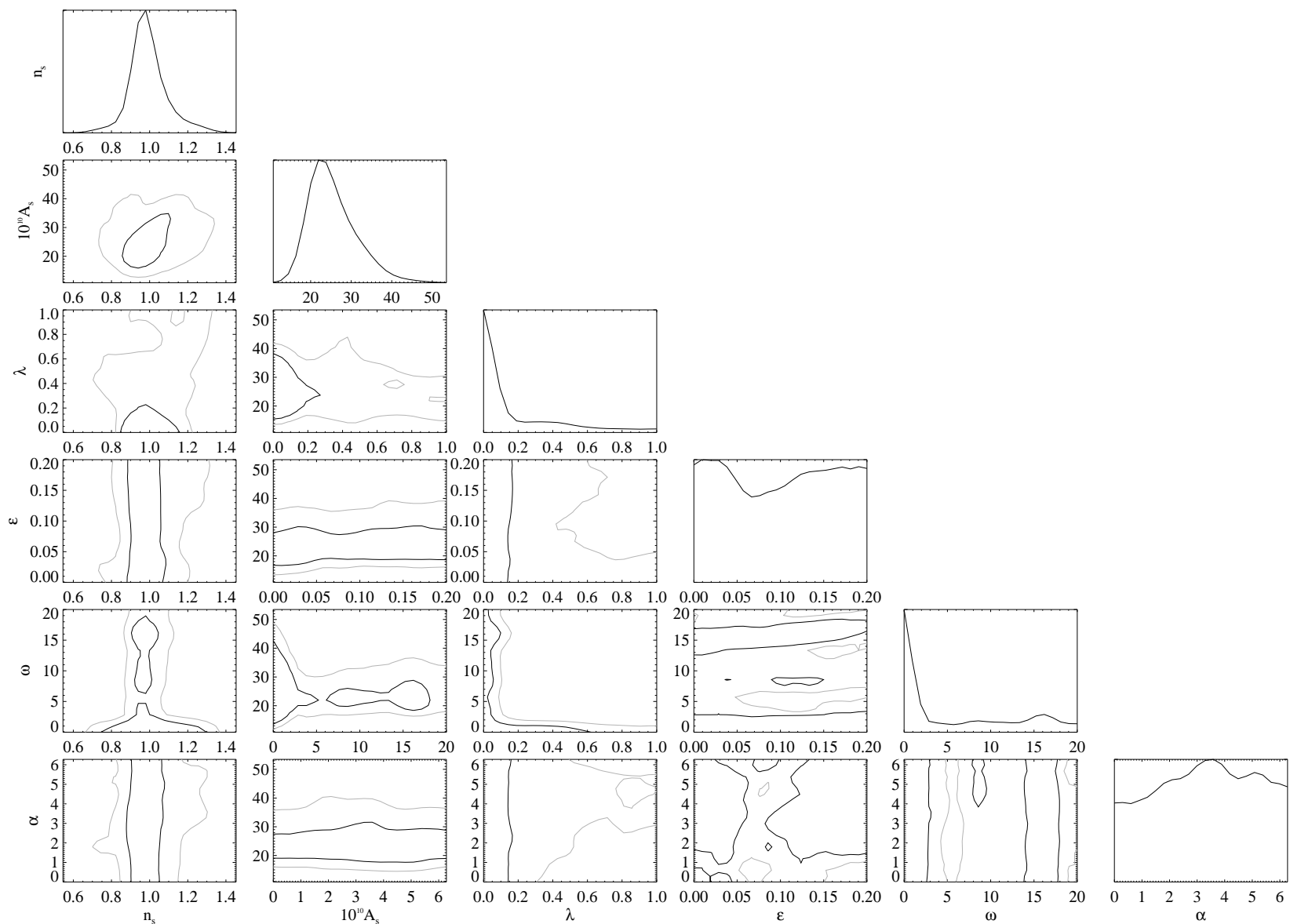

FIG. 2: WMAP likelihood contours for parameters describing the primordial power spectrum. The diagonals show the marginalized likelihoods as functions of the individual parameters. The off-diagonals show the two-dimensional likelihoods after marginalizing over the other parameters. Solid lines denote the $1 \sigma$ contours, and the gray lines the $2 \sigma$ contours.

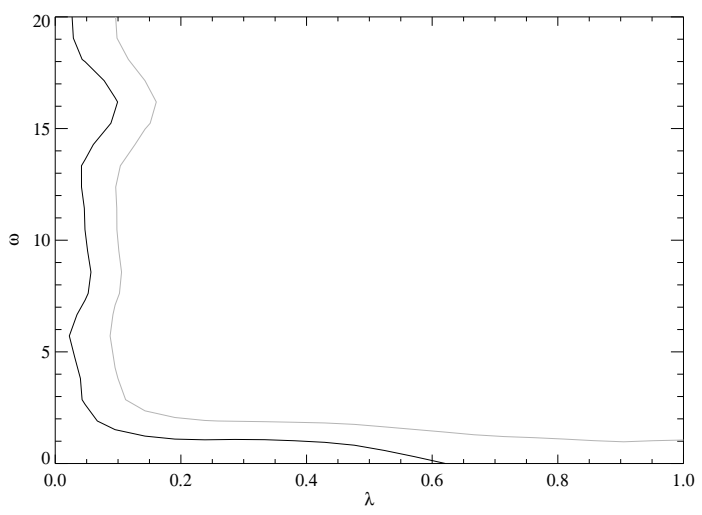

FIG. 3: Marginalized likelihood plot for the parameters $\lambda$ and $\omega$, with the solid curve denoting the $1 \sigma$ contour and the gray curve the $2 \sigma$ contour. The contours curve around the $\lambda=0$ and $\omega=0$ lines and exhibit the degeneracy between $\lambda=0$ and $\omega=0$ models. 


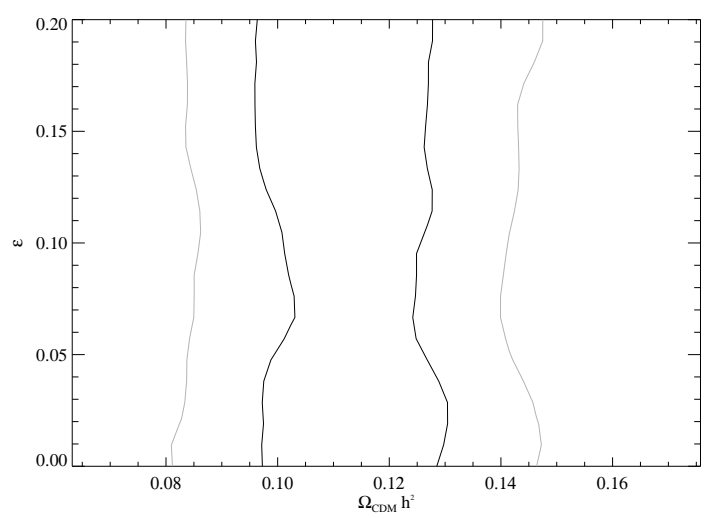

FIG. 4: Likelihood contours for $\epsilon$ vs. $\Omega_{\mathrm{CDM}} h^{2}$. There are no measurable correlations between $\epsilon$ and $\Omega_{\mathrm{CDM}} h^{2}$.
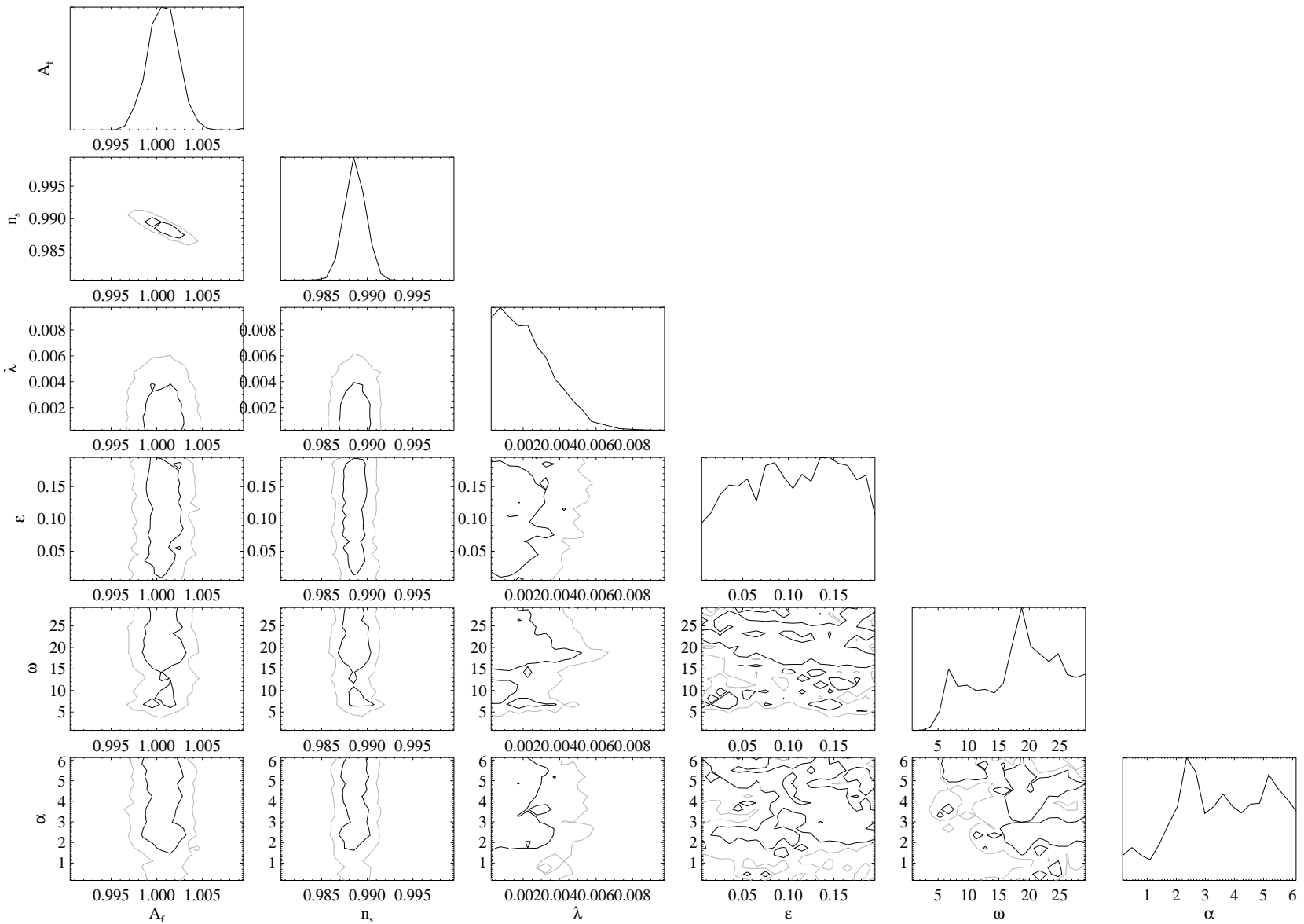

FIG. 5: $1 \sigma$ and $2 \sigma$ likelihood contours from a cosmic variance limited experiment, with a simulated data set containing no oscillations. The gray curved denote $2 \sigma$ contours. The diagonals show the marginalized likelihoods as functions of the individual parameters. 


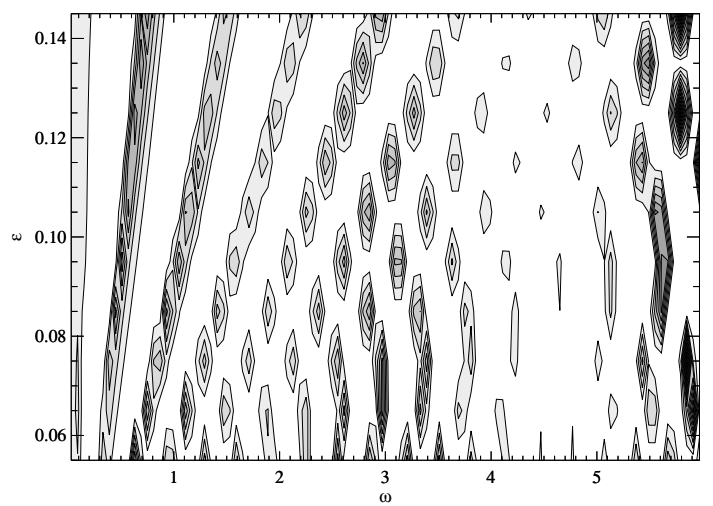

FIG. 6: The likelihood function, computed on a grid of $\omega$ and $\epsilon$, holding the other variables fixed at $\lambda=0.002, \alpha=0$. The curves are iso-likelihood contours, the levels evenly spaced between minimum and maximum, with the darker spots having higher likelihood. Near $\omega=5$, the local maxima seem to be lower than elsewhere, possibly accounting for the fact that the MCMC rules out $\omega<6$.

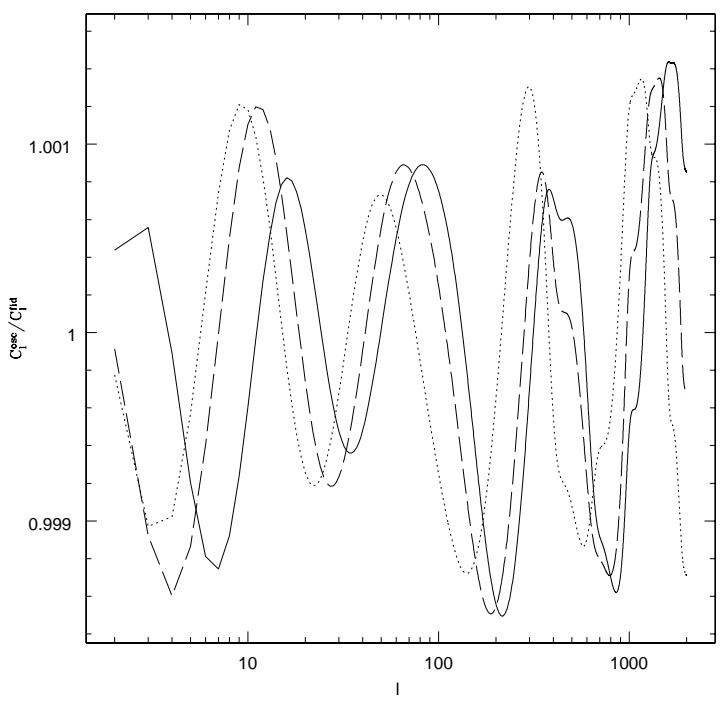

FIG. 7: Ratio of the oscillatory $C_{l}$ 's to a fiducial $C_{l}$ without oscillations, plotted for three values of $\epsilon$. Here, $\omega=4.4$, and the phase is fixed to be zero. The solid curve denotes $C_{l}$ 's with $\epsilon=0.08$, the dotted curve denotes $\epsilon=0.1$, and the dashed curve denotes $\epsilon=0.12$. Note the overall coincidence of the extremum near $l=200$, where the first acoustic peak lies. 